Research Article

\title{
The Study of a Wealth Distribution Model with a Linear Collision Kernel
}

\author{
Xia Zhou ${ }^{1},{ }^{1}$ Kaili Xiang, ${ }^{1}$ and Rongmei Sun ${ }^{2}$ \\ ${ }^{1}$ School of Economic Mathematics, Southwestern University of Finance and Economics, Chengdu 611130, China \\ ${ }^{2}$ School of Big Data Application and Economics, Guizhou University of Finance and Economics, Guiyang 550025, China \\ Correspondence should be addressed to Xia Zhou; zhouxia@smail.swufe.edu.cn
}

Received 6 May 2021; Revised 18 June 2021; Accepted 29 August 2021; Published 13 September 2021

Academic Editor: Serdar Ulubeyli

Copyright (c) 2021 Xia Zhou et al. This is an open access article distributed under the Creative Commons Attribution License, which permits unrestricted use, distribution, and reproduction in any medium, provided the original work is properly cited.

The wealth substitution rate, which describes the substitution relationship between agents' investment in wealth, is introduced into the collision kernel of the Boltzmann equation to study wealth distribution. Using the continuous trading limit, the Fokker-Planck equation is derived and the steady-state solution is obtained. The results show that the inequality of wealth distribution decreases as the wealth substitution rate increases under certain assumptions. The wealth distribution has a bimodal shape if the wealth substitution rate does not equal one.

\section{Introduction}

Since Pareto [1] discovered that wealth distribution in stable economy presents power law characteristics, the research on wealth distribution in multiagent society has been widely concerned by scholars, including economists, mathematicians, and physicists. Scholars apply different empirical studies to illustrate that the wealth distribution in the highincome range follows Pareto distribution, while in bulk ranges, it follows a Gibbs distribution, a Gamma distribution, or a log-normal distribution [2]. These results inspire them to find a theory to explain the universality of wealth distribution [3-5]. In particular, employing the kinetic theory of rarefied gases to consider wealth distribution in closed systems can better explain the fat tail of wealth distribution in the economy [5-9].

The kinetic model of wealth distribution depends on binary transactions. Chakraborti and Chakrabarti [10] introduce agents' fixed saving rate into binary transactions. Chatterjee et al. [9] discuss individual saving propensity. Cordier et al. [7] combine agents' saving propensity with risks and investigate the formation of the Pareto tail. Düring and Toscani [11] assume that there are two distinct types of agents, some of which tend to have higher saving propensity and some tend to have a lower saving propensity. The results show that wealth distribution presents a bimodal shape. The binary transaction in [8] contains knowledge, which affects saving propensity and risks. The results illustrate that knowledge is one of the reasons for the Pareto tail formation. In [6], taxation is introduced into binary transactions, and a suitable redistribution operator is constructed. The results in [6] confirm that taxation and redistribution policy modify the Pareto index, which means that taxation and redistribution policy can adjust the gap between rich and poor.

Although the wealth distribution model with binary transactions analyzes the Pareto tail formation, many scholars use the Maxwell-type collision kernel (constant collision kernel) when constructing a kinetic model. The advantage of choosing the Maxwell-type collision kernel makes the kinetic model easy to handle but ignores certain main aspects of human behavior, which may make the model deviate from practical problems. As stated in Furioli et al. [12], in the economic environment, the Maxwell-type collision kernel indicates that an agent with zero wealth or extremely small wealth can participate in transactions, leading to a net loss of market funds (excluding risks), and it may be difficult for agents to trade with such agents. Therefore, Furioli et al. [12] modify the Maxwell-type collision kernel into a variable collision kernel (also called nonMaxwell-type collision kernel). 
Inspired by the works in [12], we introduce a new kind of collision kernel, which describes two characteristics of human behavior. Under the market trading rule, agents are usually unwilling to trade with agents with zero wealth or extremely small wealth, which leads to a net loss of market funds. Consequently, the result of their mutual choice is that their wealth should be greater than or equal to a minimum value, which is the minimum wealth that the other party is willing to trade with. The realization of the transaction (or investment) in the market is due to the fact that both parties of the transaction (or investment) with their own requirements have reached an agreement on the transaction conditions. We assume that both parties finally trade with a collision frequency depicting substitutive characteristics of investing wealth. Hence, our collision kernel is

$$
K(v, w)=\alpha v+\beta w,
$$

where $0<\alpha \leq 1$ and $0<\beta \leq 1$ are constants. $\alpha$ and $\beta$ denote the proportion of wealth invested by agents with wealth $v$ and $w$, respectively. $(\beta / \alpha)$ is called wealth substitution rate, meaning that, under a certain collision frequency, agents with wealth $w$ invest one more unit of wealth, and agents with wealth $v$ can invest less $(\beta / \alpha)$ unit of wealth. Similarly, $(\alpha / \beta)$ is the wealth substitution rate of agents with wealth $v$ to agents with wealth $w$. The collision kernel with form (1) is called linear collision kernel.

The linear collision kernel (1) is not only different from the Maxwell-type collision kernel but also different from the collision kernel used in [12]. Indeed, if $K(v, w)$ is constant, it is the Maxwell-type collision kernel $[6-8,11,13,14]$, which enables agents with zero wealth or extremely small wealth in the market to participate in trading. If $K(v, w)=\kappa(v w)^{\delta}, 0<\delta \leq 1$, it is the variable collision kernel [12], which makes the agents participating in the transaction need to have a certain amount of wealth. The linear collision kernel (1) considers the rationality of agents with a certain amount of wealth participating in transactions. However, this selection brings some difficulties to our subsequent discussions. For example, compared with Maxwell-type collision kernel, the evolution of moment is different and compared with the variable collision kernel in [12], the structure of steady-state solution derived from the Fokker-Planck equation is more complex.

The main contribution of this paper is to employ the linear collision kernel (1) to discuss the influence of wealth substitution rate. Formulating the linear Boltzmann equation and seeking the asymptotic Fokker-Planck equation, we obtain the steady-state solution. The results show that the inequality of wealth distribution becomes small as the wealth substitution rate increases if $(\beta / \alpha) \neq 1$, and the wealth substitution rate has no effect on the inequality of wealth distribution when $(\beta / \alpha)=1$. We obtain that the influence of saving propensity and market risk on wealth distribution is consistent with the Maxwell-type collision kernel. The bilinear kinetic model exhibits that the wealth distribution presents a bimodal shape if $(\beta / \alpha) \neq 1$. In addition, when the collision frequency is a certain value, the wealth distribution inequality of one group of agents decreases, while the wealth distribution inequality of another group increases.
The rest of this paper is organized as follows. In Section 2 , the kinetic model with linear collision kernel is constructed and the uniformly bounded moment is discussed. In Section 3, using the continuous trading limit, we derive the asymptotic Fokker-Planck equation and study the largetime behavior of its solution. In Section 4, we build bilinear kinetic equations to discuss the wealth distribution of agents from different groups and obtain steady-state solutions. The conclusion is given in Section 5.

\section{Kinetic Model with Linear Collision Kernel}

As mentioned in the introduction, the evolution model of wealth distribution in multiagent society is mainly based on the kinetic theory of rarefied gases. The master equations of the Boltzmann type constructed by the theory are called kinetic equations, which describe the evolution of some characteristics of agents, such as wealth, knowledge, and opinions (see $[7,8,15]$ ).

According to the classical kinetic theory [5], we assume that the population of agents is homogeneous in terms of personal wealth and indistinguishable, which indicates that the agent's state at any time $t \geq 0$ is completely characterized by wealth $w \geq 0$. Hence, the state of the agent is wholly represented by the unknown distribution function (or density function) $f(w, t)$, where the wealth $w \in R_{+}$and the time $t \geq 0$. Here, the specific meaning of the distribution function $f(w, t)$ is that, for a given agent population and interval $R_{+}$, the integral $\int_{R_{+}} f(w, t) \mathrm{d} w$ denotes the number of agents who own wealth $w \in R_{+}$at time $t \geq 0$. It is generally assumed that the distribution function is normalized to one; that is,

$$
\int_{R_{+}} f(w, t) \mathrm{d} w=1
$$

The evolution of the distribution function depends on the fact that the existence of transactions in the market leads agents to constantly update their amounts of wealth $w$ in each trade. To be consistent with the classical kinetic theory of rarefied gases, we always regard a single update of the quantity $w$ as an interaction.

2.1. Kinetic Modeling. Following the kinetic theory [5], we construct kinetic equations from two aspects. Firstly, we determine the transaction rule between agents. To highlight the characteristics of the collision kernel (1), we consider a linear transaction rule. This choice not only does not change the structure of the final equilibrium but also clarifies the relationship between the interaction parameters and the equilibrium shape in a relatively simple way. The binary transaction in [7] considers the main aspects of human behavior. One is saving propensity, which describes that an agent does not exchange all his/her wealth in a transaction, ensuring keeping a small part of his/her wealth after each transaction. The other one is randomness, which depicts that the amount of wealth change is uncertain due to the existence of market risks. Thus, we introduce the save propensity and randomness into the linear transaction rule; that is, the 
agent with wealth $w$ trades in the market, and the wealth $w_{*}$ after trading is

$$
w_{*}=(1-\lambda) w+\lambda v+\eta w
$$

where constant $0<\lambda \ll 1$ denotes trading rate and $\eta$ is a random variable with mean zero and variance $\sigma$. To guarantee that the amount of wealth after trading is not negative, we assume that $\eta \geq \lambda-1 . \eta \leq \lambda_{*}<+\infty$, meaning that the random returns gained by agents in each trade are finite.

In (3), the first item on the right-hand side indicates the remaining wealth after agents who trade in the market take out $\lambda w$ part of their wealth to participate in the transaction, so $\lambda$ describes the agent's saving propensity. The second item denotes the wealth obtained by the agent after participating in the market transaction, where $v \in R_{+}$denotes the wealth owned by agents in the market with a certain distribution $h(v)$. The third item is the obtained wealth due to risks in the market.

Next, similar to the collision frequency between molecules in the classical kinetic theory of rarefied gases, we determine the transaction frequency between agents. In many previous works, this transaction frequency is a constant (see $[6-8,11,13,14])$. Inspired by these works [16-18], we mainly consider two behaviors of agents. First, under the linear transaction rule (3), as mentioned in [12], agents in the market are usually unwilling to trade with agents with zero wealth or extremely small wealth, which leads to a net loss of market funds. Consequently, the result of their mutual choice is that their respective wealth should be greater than or equal to a minimum value, which is the minimum wealth that the other party is willing to trade with. Second, the realization of the transaction (or investment) in the market is due to the fact that both parties of the transaction (or investment) with their own requirements have reached an agreement on the transaction conditions. We assume that both parties finally trade with a collision frequency depicting substitutive characteristics of investing wealth. The collision kernel that conforms to the characteristics of these two behaviors is equation (1).

Based on the above analysis, the precise interpretation of collision kernel (1) is as follows. On the one hand, agents with wealth $v$ are willing to trade with agents with wealth $w$ only when the wealth of agents with wealth $w$ meets the condition $w \geq w_{0}>0$ ( $w_{0}$ is the minimum amount of wealth accepted by agents with wealth $v$ to participate in the transaction), vice versa. Therefore, in Figure 1, the area where the upper part of half-line $B C$ overlaps with the right part of half-line $B A$ denotes that two types of agents may have transactions. On the other hand, the collision kernel measures the probability of the interaction $(v, w)$. Consequently, when the two parties trade at a collision frequency with substitutive characteristics of investing wealth, the area below segment $A C$ in Figure 1 is the possible trading region. To sum up, the area enclosed by triangle $A B C$ in Figure 1 is the feasible domain where two types of agents trade in the market.

Using liner collision kernel (1) and linear transaction rules (3), we obtain that the distribution function $f(w, t)$ of

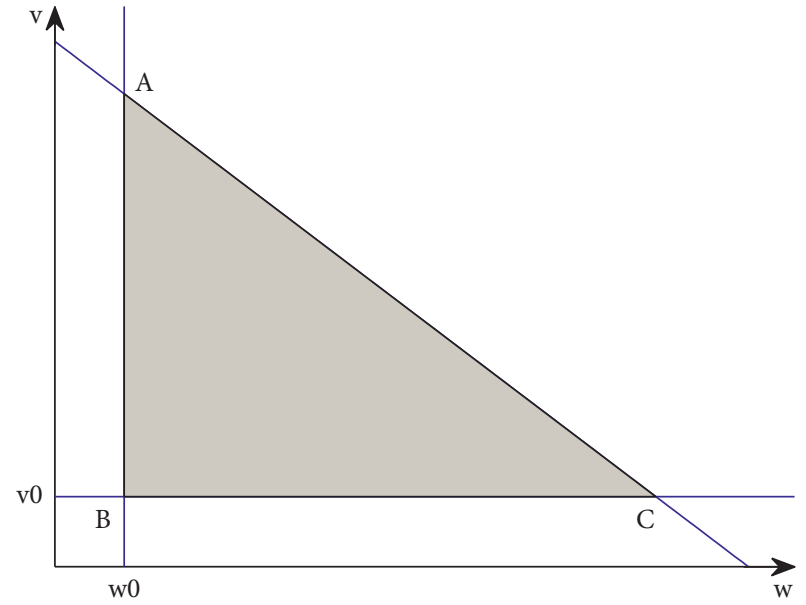

Figure 1: Graph of functions $v=v_{0}(>0), w=w_{0}(>0)$, and $v=(1 / \alpha)-(\beta / \alpha) w$.

agents follows, for any smooth function $\varphi(w)$ (the observable quantities), the linear integrodifferential equation

$$
\begin{aligned}
\frac{\mathrm{d}}{\mathrm{d} t} \int_{R_{+}} \varphi(w) f(w, t) \mathrm{d} w= & \left\langle\int _ { R _ { + } ^ { 2 } } K ( v , w ) \left(\varphi\left(w_{*}\right)\right.\right. \\
& -\varphi(w)) h(v) f(w, t) \mathrm{d} v \mathrm{~d} w\rangle,
\end{aligned}
$$

where function $h(v)\left(v \in R_{+}\right)$is the wealth distribution owned by agents in the market and $K(v, w)$ is the collision kernel defined by (1). The notation $\langle\cdot\rangle$ denotes mathematical expectation, which is mainly due to the existence of random variable $\eta$ in (3). For more details of utilizing the linear kinetic model to study wealth distribution, the reader is referred to [19].

The collision kernel (1) brings difficulties to analyze wealth distribution. An example in the following subsection will state the difficulties.

2.2. Uniformly Bounded Moments. As stated in the classical kinetic theory [5], the moments of the solution of equation (4) remain bounded in time, which is very important for analyzing the evolution of wealth distribution. To avoid unnecessary difficulties, we assume that the first four moments of $h(v)$ are bounded; namely,

$$
M_{i}=\int_{R_{+}} v^{i} h(v) \mathrm{d} v<\infty, \quad i=1,2,3,4 .
$$

The $i$-th moment of $f(w, t)$ is denoted by

$$
m_{i}(t)=\int_{R_{+}} w^{i} f(w, t) \mathrm{d} w, \quad i=1,2, \ldots, n .
$$

From equation (4), we obtain the conservation of mass. Indeed, choosing $\varphi(w)=1$ in equation (4) yields $\int_{R_{\mathrm{p}}} f(w, t) \mathrm{d} w=1$. If the initial function $f(w, 0)=f_{0}(w)$ is a probability density function, then $f(w, t)$ is still a probability density function at each subsequence time. 
Choosing $\varphi(w)=w$ in (4), we get the evolution of the mean value

$$
\frac{\mathrm{d} m_{1}(t)}{\mathrm{d} t}=\left\langle\int_{R_{+}^{2}}(\alpha v+\beta w)\left(w_{*}-w\right) h(v) f(w, t) \mathrm{d} v \mathrm{~d} w\right\rangle,
$$

and (3) implies that

$$
\left\langle w_{*}-w\right\rangle=\lambda(v-w) .
$$

Substituting (8) into (7) yields

$$
\begin{aligned}
\frac{\mathrm{d} m_{1}(t)}{\mathrm{d} t} & =\lambda \int_{R_{+}^{2}}(\alpha v+\beta w)(v-w) h(v) f(w, t) \mathrm{d} v \mathrm{~d} w \\
& =\lambda \alpha M_{2}+\lambda(\beta-\alpha) M_{1} \int_{R_{+}} w f(w, t) \mathrm{d} w \\
& -\lambda \beta \int_{R_{+}} w^{2} f(w, t) \mathrm{d} w .
\end{aligned}
$$

According to Jensen's inequality, we have

$$
\int_{R_{+}} w^{2} f(w, t) \mathrm{d} w \geq\left(\int_{R_{+}} w f(w, t) \mathrm{d} w\right)^{2} .
$$

Using this result, the evolution of the mean value becomes

$$
\frac{\mathrm{d} m_{1}(t)}{\mathrm{d} t} \leq \lambda \alpha M_{2}+\lambda(\beta-\alpha) M_{1} m_{1}(t)-\lambda \beta m_{1}(t)^{2}
$$

From (11), we have

$$
m_{1}(t) \leq \frac{x_{1}-x_{2} C_{1} e^{-\lambda C_{2} t}}{1-C_{1} e^{-\lambda C_{2} t}}
$$

where

$$
\begin{aligned}
& x_{1}=\frac{(\beta-\alpha) M_{1}+C_{2}}{2 \beta}, \\
& x_{2}=\frac{(\beta-\alpha) M_{1}-C_{2}}{2 \beta}, \\
& C_{1}=\frac{m_{1}(0)-x_{1}}{m_{1}(0)-x_{2}} \\
& C_{2}=\sqrt{(\beta-\alpha)^{2} M_{1}^{2}+4 \alpha \beta M_{2}} .
\end{aligned}
$$

Inequality (12) means that the mean value is uniformly bounded if its initial value is bounded. yields

Similarly, we consider the second moment. Using (3)

$$
\left\langle w_{*}^{2}-w^{2}\right\rangle=\left(\lambda^{2}+\sigma-2 \lambda\right) w^{2}+\lambda^{2} v^{2}+2 \lambda(1-\lambda) v w .
$$

The evolution of the second moment satisfies

$$
\begin{aligned}
\frac{\mathrm{d} m_{2}(t)}{\mathrm{d} t}= & {\left[\alpha\left(\lambda^{2}+\sigma-2 \lambda\right)+2 \beta \lambda(1-\lambda)\right] M_{1} \int_{R_{+}} w^{2} f(w, t) \mathrm{d} w } \\
& +\left[\beta \lambda^{2}+2 \alpha \lambda(1-\lambda)\right] M_{2} \int_{R_{+}} w f(w, t) \mathrm{d} w+\alpha \lambda^{2} M_{3} \\
& +\beta\left(\lambda^{2}+\sigma-2 \lambda\right) \int_{R_{+}} w^{3} f(w, t) \mathrm{d} w .
\end{aligned}
$$

If constants $\lambda$ and $\sigma$ satisfy

$$
\lambda^{2}+\sigma-2 \lambda<0
$$

letting $\left(\left(x_{1}-x_{2} C_{1} e^{-\lambda C_{2} t}\right) /\left(1-C_{1} e^{-\lambda C_{2} t}\right)\right)=D_{1}$ and utilizing Jensen's inequality, we obtain

$$
\begin{aligned}
\frac{\mathrm{d} m_{2}(t)}{\mathrm{d} t} \leq & {\left[\alpha\left(\lambda^{2}+\sigma-2 \lambda\right)+2 \beta \lambda(1-\lambda)\right] M_{1} m_{2}(t) } \\
& +\left[\beta \lambda^{2}+2 \alpha \lambda(1-\lambda)\right] M_{2} D_{1} \\
& +\beta\left(\lambda^{2}+\sigma-2 \lambda\right) D_{1}^{3}+\alpha \lambda^{2} M_{3} .
\end{aligned}
$$

Consequently, inequality (17) yields

$$
m_{2}(t) \leq m_{2}(0) e^{C_{3} t}+\frac{C_{4}}{C_{3}}\left(e^{C_{3} t}-1\right),
$$

where

$$
\begin{aligned}
& C_{3}=\left[\alpha\left(\lambda^{2}+\sigma-2 \lambda\right)+2 \beta \lambda(1-\lambda)\right] M_{1}, \\
& C_{4}=\left[\beta \lambda^{2}+2 \alpha \lambda(1-\lambda)\right] M_{2} D_{1}+\beta\left(\lambda^{2}+\sigma-2 \lambda\right) D_{1}^{3}+\alpha \lambda^{2} M_{3} .
\end{aligned}
$$

Inequality (18) implies that the second moment remains uniformly bounded if its initial value is bounded.

Using tedious calculations, it is deduced that as long as the initial moment of order $n>2$ is bounded and the coefficient of the higher-order term of wealth variable $w$ in expression $\left\langle w_{*}^{n}-w^{n}\right\rangle$ is negative (i.e., $\left.\left\langle(1-\lambda+\eta)^{n}\right\rangle-1<0\right)$, then the moment of order $n>2$ is uniformly bounded.

\section{The Fokker-Planck Equation}

Generally speaking, finding the analytical solution of equation (4) is difficult. A feasible way is to study its asymptotic behavior by means of continuous trading limit. Similar asymptotic analysis can be found in $[7,11,13,14]$ for the kinetic model of wealth distribution in multiagent society.

We assume that microscopic interaction (3) causes a slight mean change of the wealth by the scaling

$$
\begin{aligned}
& \lambda \longrightarrow \varsigma \lambda, \\
& \eta \longrightarrow \sqrt{\varsigma} \eta,
\end{aligned}
$$

where $\varsigma$ is a positive constant and satisfies $\varsigma \ll 1$. Note that the selection of the scaling has ensured that inequality (16) holds. The scaling has a small impact on the evolution of mean wealth (9); namely, 


$$
\frac{\mathrm{d} m_{1}(t)}{\mathrm{d} t}=\varsigma \lambda \int_{R_{+}^{2}}(\alpha v+\beta w)(v-w) h(v) f(w, t) \mathrm{d} v \mathrm{~d} w .
$$

Let $t \longrightarrow \varsigma \tau$; then $f(w, t)=g(w, \tau)$. In this case, we have $f_{0}(w)=g_{0}(w)$, and the evolution of mean wealth follows

$$
\frac{\mathrm{d} m_{1}(\tau)}{\mathrm{d} \tau}=\lambda \int_{R_{+}^{2}}(\alpha v+\beta w)(v-w) h(v) g(w, \tau) \mathrm{d} v \mathrm{~d} w .
$$

Comparing (9) and (22), it is observed that the scaling parameter $\varsigma$ does not affect the evolution of the mean value of $f(w, t)$.

Under the condition $\varsigma^{2} \lambda^{2}+\varsigma \sigma-2 \varsigma \lambda<0$, the evolution of the second moment is as follows:

$$
\begin{aligned}
\frac{\mathrm{d} m_{2}(\tau)}{\mathrm{d} \tau} & =\frac{1}{\varsigma}\left\langle\int_{R_{+}^{2}}(\alpha v+\beta w)\left(w_{*}^{2}-w^{2}\right) h(v) g(w, \tau) \mathrm{d} v \mathrm{~d} w\right\rangle \\
& =\int_{R_{+}^{2}}(\alpha v+\beta w)\left[(\sigma-2 \lambda) w^{2}+2 \lambda v w\right] h(v) g(w, \tau) \mathrm{d} v \mathrm{~d} w+R(\tau)
\end{aligned}
$$

where

$$
R(\tau)=c \lambda^{2} \int_{R_{+}^{2}}(\alpha v+\beta w)(v-w)^{2} h(v) g(w, \tau) \mathrm{d} v \mathrm{~d} w .
$$

In (23), the remainder $R(\tau)$ vanishes with $\varsigma \longrightarrow 0$. Therefore, the evolution of the second moment is independent of the scaling parameter $\varsigma$.

Now, we apply the simultaneous scaling of interaction parameters to deduce the evolution of a general observable quantity. Combining (3) and (20), it has

$$
\begin{aligned}
\left\langle w_{*}-w\right\rangle & =\varsigma \lambda(v-w), \\
\left\langle\left(w_{*}-w\right)^{2}\right\rangle & =\varsigma \sigma w^{2}-\varsigma^{2} \lambda^{2}(v-w)^{2} .
\end{aligned}
$$

For any smooth function $\varphi(w)$, expanding Taylor series $\varphi\left(w_{*}\right)$ around $\varphi(w)$, we get

$$
\left\langle\varphi\left(w_{*}\right)-\varphi(w)\right\rangle=\varsigma\left[\lambda \varphi^{\prime}(w)(v-w)+\frac{\varphi^{\prime \prime}(w)}{2} \sigma w^{2}\right]+r_{\varsigma}(v, w),
$$

where the remainder $r_{c}(v, w)$ vanishes at the order $\varsigma^{3 / 2}$ as $\varsigma \longrightarrow 0$ [20]. Substituting (26) into (4), after the time scaling, we obtain

$$
\begin{aligned}
& \frac{\mathrm{d}}{\mathrm{d} \tau} \int_{R_{+}} \varphi(w) g(w, \tau) \mathrm{d} w \\
& =\int_{R_{+}^{2}}(\alpha v+\beta w)\left[\lambda \varphi^{\prime}(w)(v-w)\right. \\
& \left.\quad+\frac{\varphi^{\prime \prime}(w)}{2} \sigma w^{2}\right] h(v) g(w, \tau) \mathrm{d} v \mathrm{~d} w \\
& \quad+\frac{1}{\varsigma} \int_{R_{+}^{2}}(\alpha v+\beta w) r_{\varsigma}(v, w) h(v) g(w, \tau) \mathrm{d} v \mathrm{~d} w .
\end{aligned}
$$

The second term on the right-hand side of (27) becomes zero if $\varsigma \longrightarrow 0$. Calculating the integral of the first term about $h(v)$, we acquire

$$
\begin{aligned}
& \frac{\mathrm{d}}{\mathrm{d} \tau} \int_{R_{+}} \varphi(w) g(w, \tau) \mathrm{d} w \\
& =\lambda \alpha M_{2} \int_{R_{+}} \varphi^{\prime}(w) g(w, \tau) \mathrm{d} w-\lambda(\alpha-\beta) M_{1} \int_{R_{+}} \varphi^{\prime}(w) w g(w, \tau) \mathrm{d} w \\
& \quad-\lambda \beta \int_{R_{+}} \varphi^{\prime}(w) w^{2} g(w, \tau) \mathrm{d} w+\frac{\alpha \sigma}{2} M_{1} \int_{R_{+}} \varphi^{\prime \prime}(w) w^{2} g(w, \tau) \mathrm{d} w \\
& \quad+\frac{\beta \sigma}{2} \int_{R_{+}} \varphi^{\prime \prime}(w) w^{3} g(w, \tau) \mathrm{d} w .
\end{aligned}
$$

Using integration by parts in (28), we derive the Fokker-Planck equation

$$
\begin{aligned}
\frac{\partial g(w, \tau)}{\partial \tau}= & \frac{\sigma}{2} \frac{\partial^{2}}{\partial w^{2}}\left[\left(\beta w+\alpha M_{1}\right) w^{2} g\right] \\
& +\lambda \frac{\partial}{\partial w}\left[\left(\beta w^{2}+(\alpha-\beta) M_{1} w-\alpha M_{2}\right) g\right] .
\end{aligned}
$$

In fact, the Fokker-Planck equation (29) is a special one of the following form:

$$
\frac{\partial g(x, \tau)}{\partial \tau}=\frac{\partial}{\partial x}\left[\frac{\partial}{\partial x}(i(x) g(x, \tau))+j(x) g(x, \tau)\right],
$$

where $x \geq 0$ and the diffusion coefficient $i(x) \geq 0$ and $i(x)=$ 0 is only obtained when $x=0$. In [21], Feller proves the existence and uniqueness of the solutions of equation (30). Thus, the solutions of the Fokker-Planck equation (29) exist and are unique.

As stated in [20], the right boundary condition (i.e., socalled no-flux boundary conditions) 


$$
\left\{\frac{\sigma}{2} \frac{\partial}{\partial w}\left[\left(\beta w+\alpha M_{1}\right) w^{2} g\right]+\left.\lambda\left[\left(\beta w^{2}+(\alpha-\beta) M_{1} w-\alpha M_{2}\right) g\right]\right|_{0} ^{+\infty}=0, \quad \tau>0\right.
$$

ensures mass conservation. In the Fokker-Planck equation (29), as $\tau \longrightarrow \infty$, we get that the steady-state solution $g_{\infty}(w)$ satisfies

$$
\frac{\sigma}{2} \frac{\partial}{\partial w}\left[\left(\beta w+\alpha M_{1}\right) w^{2} g_{\infty}(w)\right]+\lambda\left[\left(\beta w^{2}+(\alpha-\beta) M_{1} w-\alpha M_{2}\right) g_{\infty}(w)\right]=0
$$

Solving (32), we obtain

$$
g_{\infty}(w)=C_{5} w^{\left(A_{1} / \sigma\right)}\left[\alpha\left(M_{1}+\frac{\beta}{\alpha} w\right)\right]^{\left(A_{3} / \beta\right)} e^{-\left(A_{2} / \sigma w\right)},
$$

where the constant $C_{5}$ satisfies $\int_{R_{+}} g_{\infty}(w) \mathrm{d} w=1$ and

$$
\begin{aligned}
& A_{1}=-2(\lambda+\sigma)-2 \lambda \frac{\beta}{\alpha}\left(\frac{M_{2}}{M_{1}^{2}}-1\right), \\
& A_{2}=2 \lambda \frac{M_{2}}{M_{1}} \\
& A_{3}=-\beta\left[1-\frac{2 \lambda}{\sigma} \frac{\beta}{\alpha}\left(\frac{M_{2}}{M_{1}^{2}}-1\right)\right] .
\end{aligned}
$$

In (33), when $\beta=\alpha$ (i.e., $(\beta / \alpha)=1)$, the wealth substitution rate has no effect on wealth distribution. In this case, the steady-state wealth distribution is mainly affected by the saving propensity parameter $\lambda$, the market risks parameter $\sigma$, the proportion $\alpha$ of wealth investment, and the first and second moments of $h(v)$. When $\beta \neq \alpha$ (i.e., $(\beta / \alpha) \neq 1$ ), the wealth substitution rate has an impact on wealth distribution. The steady-state wealth distribution $g_{\infty}(w)$ corresponding to different values of wealth substitution rate is shown in Figure 2.

To analyze the influence of wealth substitution rate on wealth distribution, we employ the Lorentz curve defined as

$$
L(G(w))=\frac{\int_{0}^{w} g_{\infty}(z) z \mathrm{~d} z}{\int_{R_{+}} g_{\infty}(z) z \mathrm{~d} z}
$$

where $G(w)=\int_{0}^{w} g_{\infty}(z) \mathrm{d} z$ is the cumulative density function. According to the Lorentz curve, the Gini coefficient [22] is

$$
G=1-2 \int_{0}^{1} L(G(w)) \mathrm{d} w
$$

The Gini coefficient is regarded as a measure of inequality in wealth distribution, and it varies in $[0,1] . G=0$ denotes perfect equality, and $G=1$ means perfect inequality. The Lorentz curves corresponding to different wealth substitution rates are presented in Figure 2. We calculate the Gini coefficient corresponding to these Lorentz curves in
Table 1 (in fact, in our model, there exists a larger Gini coefficient than 0.5512 . This is consistent with the results of a recent study by Alfani and Schifano 2021 (https://www. oecdilibrary.org/sites/3d96efc5-en/index.html?itemId=/con tent/publication/3d96efc5-en); namely, the typical value of wealth inequality is in the range $0.52-0.88$ for the post- 1980 period. However, if we want to calculate the larger Gini coefficient, we need to choose a smaller wealth substitution rate and saving propensity and larger risk parameters). It is clearly seen that the inequality of wealth distribution decreases with the increase of the wealth substitution rate.

Under linear collision kernel, the influence of saving propensity and market risks on wealth distribution is consistent with that under Maxwell-type collision kernel. Figure 3 displays the steady-state wealth distribution and the Lorentz curve of different saving propensity parameter values. The Gini coefficient is calculated in Table 2. Clearly, the inequality of wealth distribution reduces as the saving propensity increases. The steady-state wealth distribution and the Lorentz curve of different risk parameter values are shown in Figure 4, and the Gini coefficient is given in Table 2 . It is observed that the increase of market risk leads to the increase of inequality of wealth distribution.

\section{Bilinear Kinetic Model}

In the previous section, the Boltzmann equation is constructed by a linear transaction rule and collision kernel (1), and its corresponding Fokker-Planck equation is derived by means of a continuous trading limit. This method is similarly extended to binary transactions. Binary transaction rules with saving propensity and risks have been introduced in [7]. According to the idea in [7], we consider two groups of agents with wealth $v, w$, respectively, in the market and update their amounts of wealth according to the rule

$$
\begin{gathered}
v_{*}=(1-\lambda) v+\lambda w+\eta_{1} v, \\
w_{*}=(1-\lambda) w+\lambda v+\eta_{2} w,
\end{gathered}
$$

where $\lambda$ is a constant trading rate, such that $0<\lambda \ll 1$, and $\eta_{1}, \eta_{2}$ are independent and identically distributed random variables with mean zero and variance $\sigma$, which are independent of wealth variables. Similarly, to guarantee that the wealth variables after trading are not negative, we assume $\eta_{1}, \eta_{2} \geq \lambda-1$. 


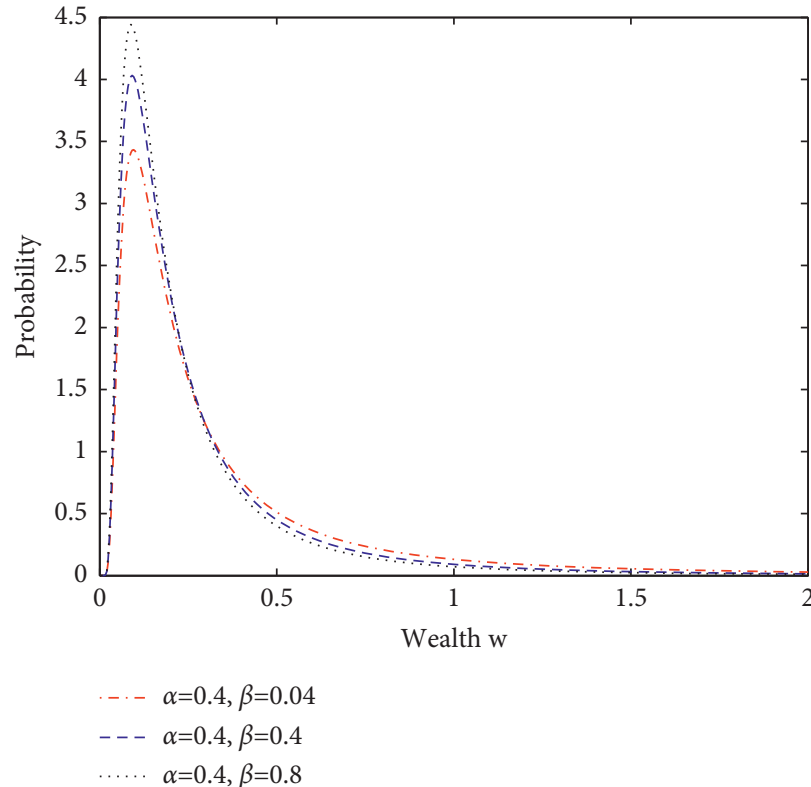

(a)

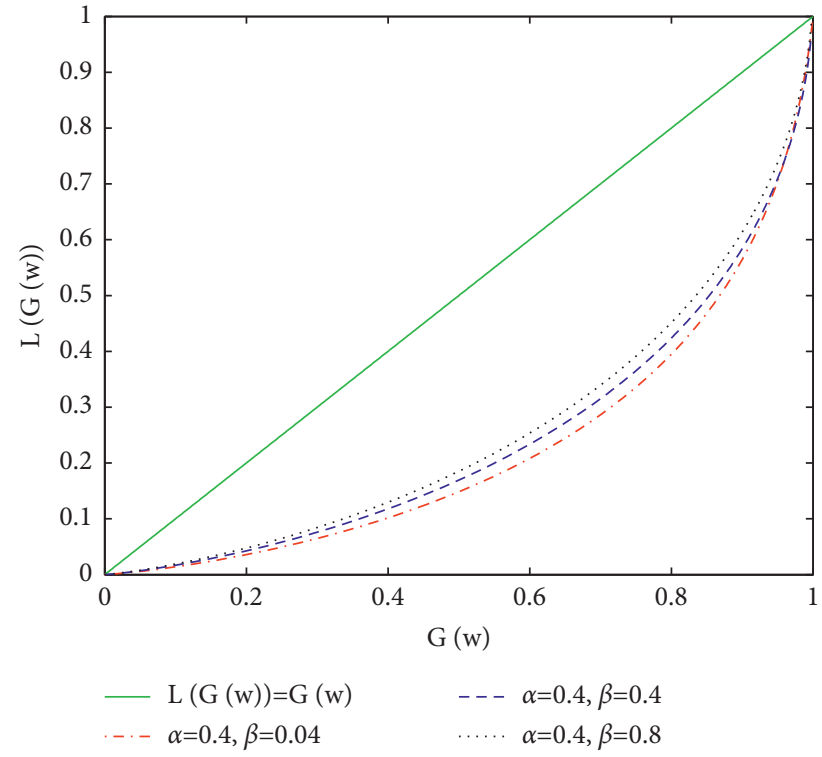

(b)

FIGURE 2: Steady-state probability $g_{\infty}(w)$ (a) and corresponding Lorentz curves (b) for $\lambda=0.05, \sigma=0.5, M_{1}=1$, and $M_{2}=1.05$ and different $(\beta / \alpha)$.

TABLE 1: Gini coefficient for the steady-state wealth distribution with different wealth substitution rates.

\begin{tabular}{lccc}
\hline$\alpha$ & $\beta$ & $\beta / \alpha$ & Gini coefficient \\
\hline 0.4 & 0.04 & 0.1 & 0.5512 \\
0.4 & 0.4 & 1 & 0.5090 \\
0.4 & 0.8 & 2 & 0.4922 \\
\hline
\end{tabular}

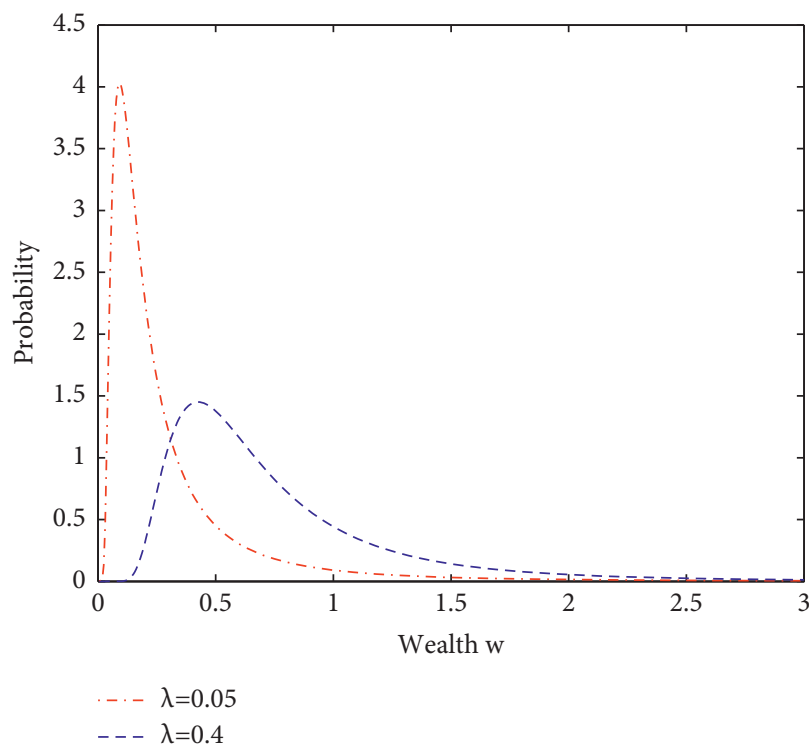

(a)

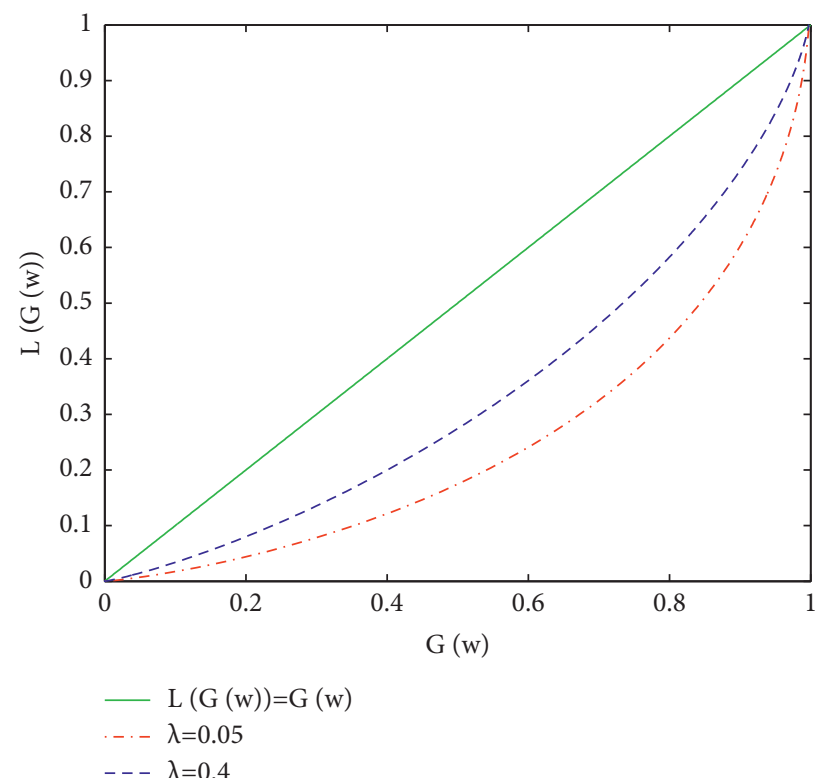

(b)

FIGURE 3: Steady-state probability $g_{\infty}(w)$ (a) and corresponding Lorentz curves (b) for $\alpha=0.4, \beta=0.4, \sigma=0.5, M_{1}=1$, and $M_{2}=1.05$ and different $\lambda$. 
TABLE 2: Gini coefficient for the steady-state wealth distribution with different wealth substitution rates.

\begin{tabular}{lccr}
\hline$\lambda$ & Gini coefficient & $\sigma$ & Gini coefficient \\
\hline 0.05 & 0.5090 & 0.1 & 0.3812 \\
0.4 & 0.3384 & 0.5 & 0.5090 \\
\hline
\end{tabular}

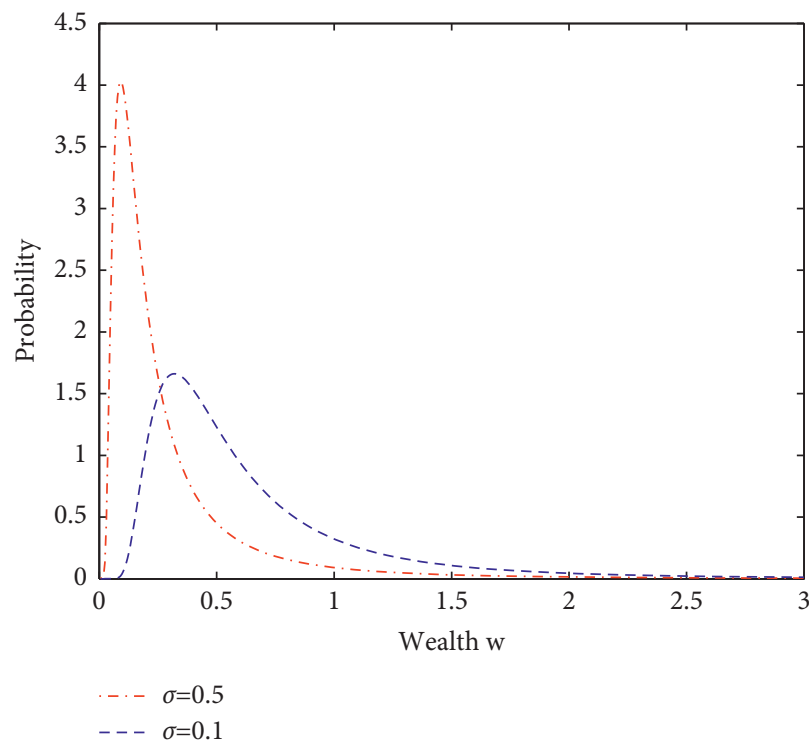

(a)

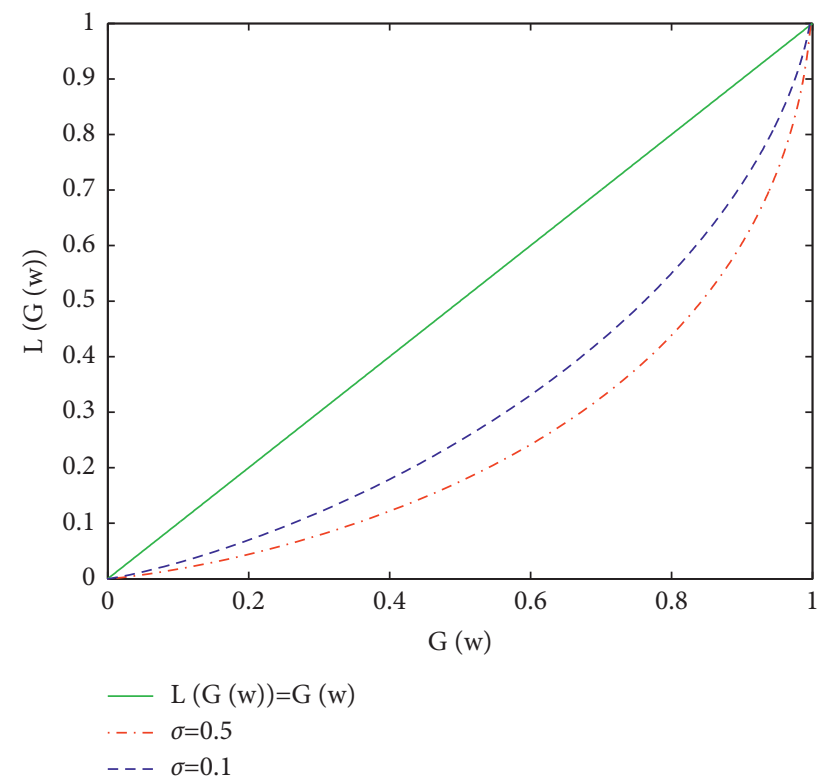

(b)

FIGURE 4: Steady-state probability $g_{\infty}(w)$ (a) and corresponding Lorentz curves (b) for $\alpha=0.4, \beta=0.4, \lambda=0.05, M_{1}=1$, and $M_{2}=1.05$ and different $\sigma$.

Obviously, (37) yields

$$
\left\langle v_{*}+w_{*}\right\rangle=v+w
$$

and this means that total wealth is conserved in a statistical sense.

According to the collision kernel (1), the proportion of wealth invested by agents with wealth $v$ is $\alpha$, and that of agents with wealth $w$ is $\beta$. $\alpha=\beta$ means that there is only one kind of agent in the market. $\alpha \neq \beta$ indicates that there are two groups of agents in the market. Let $f_{1}(w, t)$ and $f_{2}(w, t)$ represent the wealth distribution of agents whose investment ratio is $\alpha$ and $\beta$, respectively. The total wealth distribution is $f(w, t)=f_{1}(w, t)+f_{2}(w, t)$. We fix initial total wealth distribution which is unit mass; that is, $\int_{R_{+}} f(w, 0) \mathrm{d} w=1$. Following the ideas in [11], the evolution of $f_{j}(w, t)$ satisfies the bilinear kinetic equation

$$
\frac{\partial f_{j}(w, t)}{\partial t}=Q\left(f_{j}, f_{\bar{j}}\right)(w)+Q\left(f_{j}, f_{j}\right)(w), \quad j, \bar{j}=1,2 \text {, and } j \neq \bar{j},
$$

where the collision operators $Q\left(f_{j}, f_{\bar{j}}\right)(w)$ and $Q\left(f_{j}, f_{j}\right)(w)$ denote the evolution of $f_{j}(w, t)$ due to transaction between agents of different groups and the same group, respectively. To clarify the characteristics of collision kernel (1), we assume that agents of the same group do not trade. In this setting, $Q\left(f_{j}, f_{j}\right)(w)=0$; the weak form of (39) is given by

$$
\begin{aligned}
& \frac{\mathrm{d}}{\mathrm{d} t} \int_{R_{+}} \varphi(w) f_{j}(w, t) \mathrm{d} w \\
& =\frac{1}{2}\left\langle\int _ { R _ { + } ^ { 2 } } K ( v , w ) \left(\varphi\left(w_{*}\right)+\varphi\left(v_{*}\right)-\varphi(w)\right.\right. \\
& \left.\quad-\varphi(v)) f_{j}(w, t) f_{\bar{j}}(v, t) \mathrm{d} v \mathrm{~d} w\right\rangle \\
& =\left\langle\int_{R_{+}^{2}} K(v, w)\left(\varphi\left(w_{*}\right)-\varphi(w)\right) f_{j}(w, t) f_{\bar{j}}(v, t) \mathrm{d} v \mathrm{~d} w\right\rangle,
\end{aligned}
$$

where $\varphi(\cdot)$ is any smooth function, the collision kernel $K(v, w)=\alpha w+\beta v$ if $j=1$, and $K(v, w)=\beta w+\alpha v$ if $j=2$.

In (40), choosing $\varphi(w)=1$, we obtain $(\mathrm{d} / \mathrm{d} t) \int_{R_{+}} f_{j}(w, t) \mathrm{d} w=0$. This means that the solution of (40) satisfies mass conservation; that is,

$$
\begin{aligned}
& \int_{R_{+}} f_{1}(w, t) \mathrm{d} w=\int_{R_{+}} f_{1}(w, 0) \mathrm{d} w=\rho_{1}, \\
& \int_{R_{+}} f_{2}(w, t) \mathrm{d} w=\int_{R_{+}} f_{2}(w, 0) \mathrm{d} w=\rho_{2},
\end{aligned}
$$


and $\rho_{1}+\rho_{2}=1$. For any $K(v, w), \varphi(w)=w$ yields

$$
\begin{aligned}
& \frac{\mathrm{d}}{\mathrm{d} t} \int_{R_{+}} w f_{j}(w, t) \mathrm{d} w \\
& =\frac{1}{2}\left\langle\int_{R_{+}^{2}} K(v, w)\left(w_{*}+v_{*}-w-v\right) f_{j}(w, t) f_{\bar{j}}(v, t) \mathrm{d} v \mathrm{~d} w\right\rangle=0 .
\end{aligned}
$$

This indicates that the mean wealth is conserved,

$$
\begin{aligned}
& \int_{R_{+}} w f_{1}(w, t) \mathrm{d} w=m_{1,1}(0), \\
& \int_{R_{+}} w f_{2}(w, t) \mathrm{d} w=m_{1,2}(0),
\end{aligned}
$$

and the total mean wealth is $m_{1,1}(0)+m_{1,2}(0)=m_{1}(0)$, where the first subscript of $m_{i, j}$ represents the $i$-th moment of $f_{j}(w, t)$ and the second subscript represents the $j$-th class agent. It is difficult to obtain the analytical expression of high-order moment evolution. For this reason, we assume that the moment of the solution of equation (40) is bounded up to order three.

In (40), for the evolution of $f_{1}(w, t)$, applying the scaling (20) and the time scaling, using the Taylor expanding to $\varphi(w)$, we obtain the weak form of Fokker-Planck equation corresponding to equation (40)

$$
\begin{aligned}
& \frac{\mathrm{d}}{\mathrm{d} \tau} \int_{R_{+}} \varphi(w) g_{1}(w, \tau) \mathrm{d} w \\
& =\lambda \beta m_{2,2}(\tau) \int_{R_{+}} \varphi^{\prime}(w) g_{1}(w, \tau) \mathrm{d} w+\lambda(\alpha-\beta) m_{1,2}(0) \int_{R_{+}} \varphi^{\prime}(w) w g_{1}(w, \tau) \mathrm{d} w \\
& \quad-\lambda \alpha \rho_{2} \int_{R_{+}} \varphi^{\prime}(w) w^{2} g_{1}(w, \tau) \mathrm{d} w+\frac{\rho_{2} \alpha \sigma}{2} \int_{R_{+}} \varphi^{\prime \prime}(w) w^{3} g_{1}(w, \tau) \mathrm{d} w \\
& \quad+\frac{\beta \sigma m_{1,2}(0)}{2} \int_{R_{+}} \varphi^{\prime \prime}(w) w^{2} g_{1}(w, \tau) \mathrm{d} w .
\end{aligned}
$$

Making use of boundary conditions, we deduce the Fokker-Planck equation

$$
\begin{aligned}
\frac{\partial g_{1}(w, \tau)}{\partial \tau}= & \frac{\sigma}{2} \frac{\partial^{2}}{\partial w^{2}}\left[\left(\rho_{2} \alpha w+\beta m_{1,2}(0)\right) w^{2} g_{1}(w, \tau)\right] \\
& +\lambda \frac{\partial}{\partial w}\left[\left(\rho_{2} \alpha w^{2}\right.\right. \\
& \left.\left.+(\beta-\alpha) m_{1,2}(0) w-\beta m_{2,2}(\tau)\right) g_{1}(w, \tau)\right]
\end{aligned}
$$

where $m_{2,2}(\tau)=\int_{R_{+}} v^{2} g_{2}(v, \tau) \mathrm{d} v$. In the same way, we obtain the equation

$$
\begin{aligned}
\frac{\partial g_{2}(w, \tau)}{\partial \tau}= & \frac{\sigma}{2} \frac{\partial^{2}}{\partial w^{2}}\left[\left(\rho_{1} \beta w+\alpha m_{1,1}(0)\right) w^{2} g_{2}(w, \tau)\right] \\
& +\lambda \frac{\partial}{\partial w}\left[\left(\rho_{1} \beta w^{2}+(\alpha-\beta) m_{1,1}(0) w\right.\right. \\
& \left.\left.-\alpha m_{2,1}(\tau)\right) g_{2}(w, \tau)\right],
\end{aligned}
$$

where $m_{2,1}(\tau)=\int_{R_{+}} v^{2} g_{1}(v, \tau) \mathrm{d} v$.

From (45), we get the steady-state solution

$$
g_{1, \infty}(w)=C_{6} w^{\left(A_{4} / \sigma\right)}\left[\beta\left(m_{1,2}(0)+\rho_{2} \frac{\alpha}{\beta} w\right)\right]^{\left(A_{6} / \rho_{2} \alpha\right)} e^{-\left(A_{5} / \sigma w\right)},
$$

where the constant $C_{6}$ satisfies $\int_{R_{+}} g_{1, \infty}(w) \mathrm{d} w=\rho_{1}$ and

$$
\begin{aligned}
& A_{4}=-2(\lambda+\sigma)-2 \lambda \frac{\alpha}{\beta}\left(\rho_{2} \frac{m_{2,2}(\infty)}{m_{1,2}(0)^{2}}-1\right), \\
& A_{5}=2 \lambda \frac{m_{2,2}(\infty)}{m_{1,2}(0)} \\
& A_{6}=-\rho_{2} \alpha\left[1-\frac{2 \lambda}{\sigma} \frac{\alpha}{\beta}\left(\rho_{2} \frac{m_{2,2}(\infty)}{m_{1,2}(0)^{2}}-1\right)\right] .
\end{aligned}
$$

Similarly, we obtain

$$
g_{2, \infty}(w)=C_{7} w^{\left(A_{7} / \sigma\right)}\left[\alpha\left(m_{1,1}(0)+\rho_{1} \frac{\beta}{\alpha} w\right)\right]^{\left(A_{9} / \rho_{1} \beta\right)} e^{-\left(A_{8} / \sigma w\right)}
$$

where the constant $C_{7}$ satisfies $\int_{R_{+}} g_{2, \infty}(w) \mathrm{d} w=\rho_{2}$ and

$$
\begin{aligned}
& A_{7}=-2(\lambda+\sigma)-2 \lambda \frac{\beta}{\alpha}\left(\rho_{1} \frac{m_{2,1}(\infty)}{m_{1,1}(0)^{2}}-1\right), \\
& A_{8}=2 \lambda \frac{m_{2,1}(\infty)}{m_{1,1}(0)} \\
& A_{9}=-\rho_{1} \beta\left[1-\frac{2 \lambda}{\sigma} \frac{\beta}{\alpha}\left(\rho_{1} \frac{m_{2,1}(\infty)}{m_{1,1}(0)^{2}}-1\right)\right] .
\end{aligned}
$$




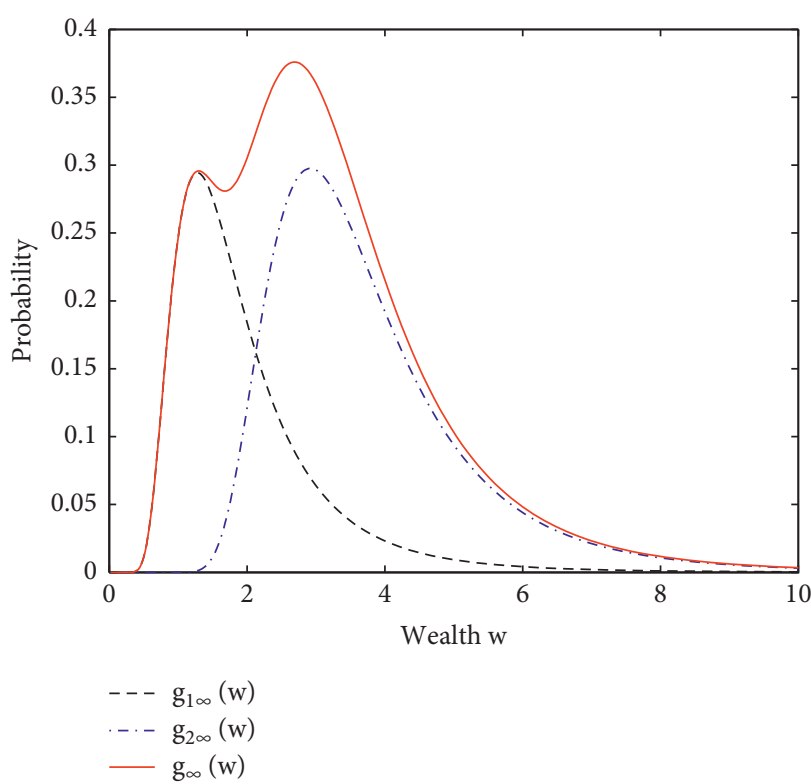

(a)

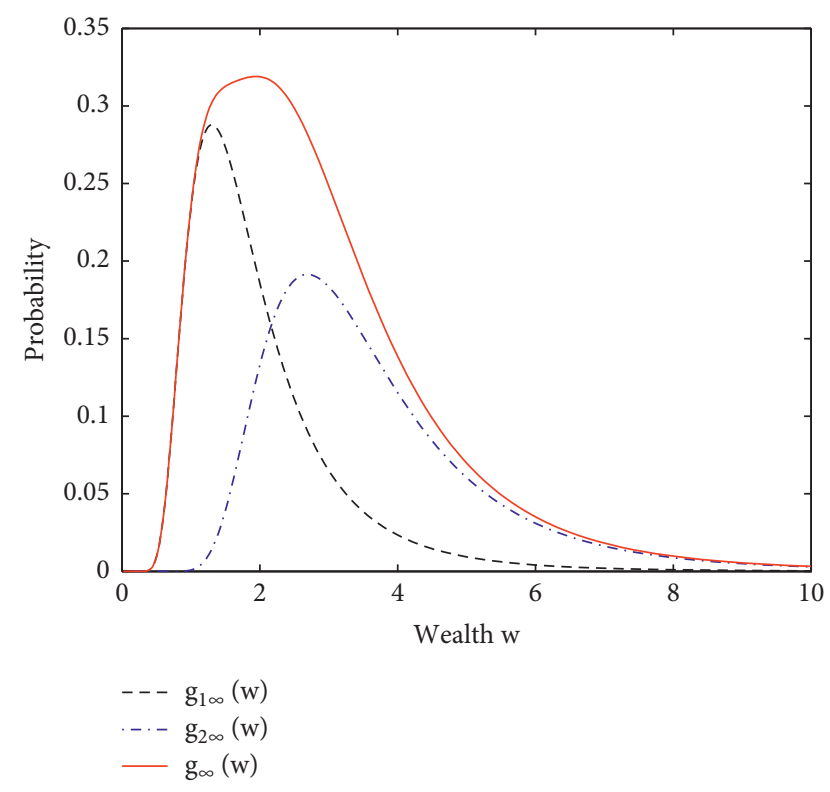

(b)

FIGURE 5: Steady-state probability $g_{1, \infty}(w), g_{2, \infty}(w)$, and $g_{\infty}(w)$ (a) for $\rho_{1}=\rho_{2}=0.5, \alpha=0.2, \beta=0.6, \lambda=0.2, \sigma=0.1, m_{1,1}(0)=1$, and $m_{1,2}(0)=3$. Steady-state probability $g_{1, \infty}(w), g_{2, \infty}(w)$, and $g_{\infty}(w)(b)$ for $\rho_{1}=\rho_{2}=0.5, \alpha=0.2, \beta=0.4, \lambda=0.2, \sigma=0.1, m_{1,1}(0)=1$, and $m_{1,2}(0)=2$.

It is seen that the structure of steady-state solutions in (47) and (49) is consistent with that in (33). Therefore, if the proportion of wealth investment of one group of agents is unchanged, the proportion of wealth investment of the other group increases, that is, the wealth substitution rate of the other group increases, and then the inequality of wealth distribution of the other group decreases. If the collision frequency is a certain value, the increase of the wealth substitution rate of one group leads to the decrease of the wealth substitution rate of the other group, meaning that the inequality of wealth distribution of one group decreases while that of the other group increases.

Taking the sum of (47) and (49) gives the total wealth distribution

$$
\begin{aligned}
g_{\infty}(w)= & C_{6} w^{\left(A_{4} / \sigma\right)}\left(\rho_{2} \alpha w+\beta m_{1,2}(0)\right)^{\left(A_{6} / \rho_{2} \alpha\right)} e^{-\left(A_{5} / \sigma w\right)} \\
& +C_{7} w^{\left(A_{7} / \sigma\right)}\left(\rho_{1} \beta w+\alpha m_{1,1}(0)\right)^{\left(A_{9} / \rho_{1} \beta\right)} e^{-\left(A_{8} / \sigma w\right) .}
\end{aligned}
$$

In (51), when $\alpha=\beta$, the wealth substitution rate does not affect the shape of total wealth distribution. When $\alpha \neq \beta$, the wealth substitution rate leads to a bimodal of total wealth distribution. Indeed, the maximum value of wealth distribution $g_{1, \infty}(w)$ arrives at

$$
\begin{aligned}
w_{1, \max } & =\frac{-\left(a_{1} \beta m_{1,2}(0)+a_{3} \rho_{2} \alpha\right)-\sqrt{\Delta}}{2 \rho_{2} \alpha\left(a_{1}+a_{2}\right)}, \\
\Delta & =\left(a_{1} \beta m_{1,2}(0)+a_{3} \rho_{2} \alpha\right)^{2}-4 \rho_{2} \alpha \beta\left(a_{1}+a_{2}\right) a_{3} m_{1,2}(0),
\end{aligned}
$$

where $a_{1}=\left(A_{4} / \sigma\right), a_{2}=\left(A_{6} / \rho_{2} \alpha\right), a_{3}=\left(A_{5} / \sigma\right)>0$, and $a_{1}+a_{2}=-3-(2 \lambda / \sigma)<0$. However, the maximum value of wealth distribution $g_{2, \infty}(w)$ arrives at

$$
\begin{aligned}
w_{2, \max } & =\frac{-\left(a_{4} \alpha m_{1,1}(0)+\rho_{1} \beta a_{6}\right)-\sqrt{\Delta}}{2 \rho_{1} \beta\left(a_{4}+a_{5}\right)}, \\
\Delta & =\left(a_{4} \alpha m_{1,1}(0)+\rho_{1} \beta a_{6}\right)^{2}-4 \alpha \beta \rho_{1}\left(a_{4}+a_{5}\right) a_{6} m_{1,1}(0),
\end{aligned}
$$

where $a_{4}=\left(A_{7} / \sigma\right), a_{5}=\left(A_{9} / \rho_{1} \beta\right), a_{6}=\left(A_{8} / \sigma\right)>0$, and $a_{4}+a_{5}=-3-(2 \lambda / \sigma)<0$. See $[11,23]$ for more details on bimodal distribution. If $\alpha \neq \beta$, then $w_{1, \max } \neq w_{2, \max }$. In this case, Figure 5(a) shows that the total wealth distribution presents a bimodal shape. However, different selections of parameters may produce a single peak shape (see Figure 5(b)).

\section{Conclusion}

In this paper, the wealth substitution rate which describes the substitution relationship between agents' investment is introduced into the collision kernel of the Boltzmann equation to study wealth distribution. By resorting to the kinetic theory of rarefied gases, we build a linear kinetic equation. Using the continuous trading limit, we derive the corresponding the Fokker-Planck equation and obtain the steady-state solution. The results show that the inequality of wealth distribution becomes small as the wealth substitution rate increases if $(\beta / \alpha) \neq 1$, and the wealth substitution rate has no effect on the inequality of wealth distribution if 
$(\beta / \alpha)=1$. In addition, we obtain that the impact of saving propensity and market risks on wealth distribution under the linear collision kernel is consistent with the Maxwelltype collision kernel. Applying a similar method, the bilinear kinetic model illustrates that when the collision frequency is a certain value, the change of wealth substitution rate leads to a decrease in the wealth distribution inequality of one group of agents and an increase in the wealth distribution inequality of another group. If $(\beta / \alpha) \neq 1$, the total wealth distribution presents a bimodal shape.

\section{Data Availability}

The data used to support the findings of this study are available from the corresponding author upon request.

\section{Conflicts of Interest}

The authors declare that there are no conflicts of interest regarding the publication of this paper.

\section{Acknowledgments}

This research was supported by the National Natural Science Foundation of China (no. 11471263) and the Fundamental Research Funds for the Central Universities (no. JBK2107111).

\section{References}

[1] V. Pareto, Cours D'economie Politique, Macmillan, Lausanne, Switzerland, 1897.

[2] B. K. Chakrabarti, A. Chakraborti, S. R. Chakravarty, and A. Chatterjee, Econophysics of Income and Wealth Distribution, Cambridge University Press, Cambridge, UK, 2013.

[3] J. Benhabib, A. Bisin, and S. Zhu, "The wealth distribution in Bewley economies with capital income risk," Journal of Economic Theory, vol. 159, pp. 489-515, 2015.

[4] C. Hu, W. Huang, and T. Xie, "The investigation of a wealth distribution model on isolated discrete time domains," Mathematical Problems in Engineering, vol. 2020, Article ID 4353025, 2020.

[5] L. Pareschi and G. Toscani, Interacting Multiagent systems: Kinetic Equations \& Monte Carlo Methods, Oxford University Press, Oxford, UK, 2013.

[6] M. Bisi, G. Spiga, and G. Toscani, "Kinetic models of conservative economies with wealth redistribution," Communications in Mathematical Sciences, vol. 7, no. 4, pp. 901-916, 2009.

[7] S. Cordier, L. Pareschi, and G. Toscani, "On a kinetic model for a simple market economy," Journal of Statistical Physics, vol. 120, no. 1-2, pp. 253-277, 2005.

[8] L. Pareschi and G. Toscani, "Wealth distribution and collective knowledge. a Boltzmann approach," Philosophical Transactions of the Royal Society A: Mathematical, Physical and Engineering Sciences, vol. 372, no. 2028, pp. 1-15, 2014.

[9] A. Chatterjee, B. K. Chakrabarti, and S. S. Manna, "Pareto law in a kinetic model of market with random saving propensity," Physica A: Statistical Mechanics and Its Applications, vol. 335, no. 1-2, pp. 155-163, 2004.
[10] A. Chakraborti and B. K. Chakrabarti, "Statistical mechanics of money: how saving propensity affects its distribution," The European Physical Journal B, vol. 17, no. 1, pp. 167-170, 2000.

[11] B. Düring and G. Toscani, "International and domestic trading and wealth distribution," Communications in Mathematical Sciences, vol. 6, no. 4, pp. 1043-1058, 2008.

[12] G. Furioli, A. Pulvirenti, E. Terraneo, and G. Toscani, "NonMaxwellian kinetic equations modeling the dynamics of wealth distribution," Mathematical Models and Methods in Applied Sciences, vol. 30, no. 4, pp. 685-725, 2020.

[13] M. Bisi, "Some kinetic models for a market economy," Bollettino dell'Unione Matematica Italiana, vol. 10, no. 1, pp. 143-158, 2017.

[14] M. Bisi, G. Spiga, and G. Spiga, “A Boltzmann-type model for market economy and its continuous trading limit," Kinetic and Related Models, vol. 3, no. 2, pp. 223-239, 2010.

[15] E. Cristiani and A. Tosin, "Reducing complexity of multiagent systems with symmetry breaking: an application to opinion dynamics with polls," Multiscale Modeling and Simulation, vol. 16, no. 1, pp. 528-549, 2018.

[16] G. Albi, L. Pareschi, and M. Zanella, "Uncertainty quantification in control problems for flocking models," Mathematical Problems in Engineering, vol. 2015, Article ID 850124, 14 pages, 2015.

[17] N. Bellomo, D. Knopoff, and J. Soler, "On the difficult interplay between life, "complexity", and mathematical sciences," Mathematical Models and Methods in Applied Sciences, vol. 23, no. 10, pp. 1861-1913, 2013.

[18] N. Bellomo, M. A. Herrero, M. A. Herrero, and A. Tosin, "On the dynamics of social conflicts: looking for the black swan," Kinetic and Related Models, vol. 6, no. 3, pp. 459-479, 2013.

[19] G. Toscani, A. Tosin, A. Tosin, and M. Zanella, "Kinetic modelling of multiple interactions in socio-economic systems," Networks and Heterogeneous Media, vol. 15, no. 3, pp. 519-542, 2020.

[20] G. Furioli, A. Pulvirenti, E. Terraneo, and G. Toscani, "FokkerPlanck equations in the modelling of socio-economic phenomena," Mathematical Models and Methods in Applied Sciences, vol. 27, no. 1, pp. 115-158, 2017.

[21] W. Feller, "The parabolic differential equations and the associated semi-groups of transformations," Annals of Mathematics, vol. 55, no. 3, pp. 468-519, 1952.

[22] C. Gini, "Measurement of inequality of incomes," The Economic Journal, vol. 31, no. 121, pp. 124-126, 1921.

[23] A. Ghosh, A. Chatterjee, J.-I. Inoue, and B. K. Chakrabarti, "Inequality measures in kinetic exchange models of wealth distributions," Physica A: Statistical Mechanics and Its Applications, vol. 451, no. 1, pp. 465-474, 2016. 